\title{
Comparison of factor loadings for anthropometric and physiometric measures among type 2 diabetic males, pre- and post-menopausal females in North Indian Punjabi population
}

\author{
Badaruddoza*, Basanti Barna, Amarjit Singh Bhanwer \\ Department of Human Genetics, Guru Nanak Dev University, Amritsar, India; *Corresponding Author: doza13@yahoo.co.in
}

Received 10 April 2010; revised 18 May 2010; accepted 23 May 2010.

\begin{abstract}
Background: The objective of the present study was to compare the relationship of anthropometric and physiometric characteristics using principal component factor analysis among three groups of type 2 diabetic subjects such as males, pre- and post-menopausal females in North Indian Punjabi population. Method: A total of 349 type 2 diabetic subjects (males 157; females 192; 88 pre- and 104 post-menopausal) were ascertained for the present study. Different anthropometric and physiometric measurements were taken. Principal component factor analysis (PCFA) was applied to identify the components which are more close to type 2 diabetes among the three groups. Results: PCFA revealed five uncorrelated components which explained $79 \%$ of the total variance among diabetic males and six unrelated components which explained $78 \%$ of the total variance among pre- and post-menopausal females. The important two factors could be identified as central obesity (factor 1) and blood pressure (factor 2) among these three groups. Conclusion: Higher clustering of obesity and blood pressures were found in diabetic males as compared to pre- and post-menopausal diabetic females in North Indian Punjabi population whereas, waist to hip ratio (WHR) has maximum loading in post-menopausal females as compared to others.
\end{abstract}

Keywords: Factor Analysis; Blood Pressure; Type 2 Diabetes; Anthropometry; Punjabi Population

\section{INTRODUCTION}

The relationships between type 2 diabetes mellitus (T2DM), anthropometric variables and blood pressures are statistically complex [1,2]. Strong inter-correlation between anthropometric and physiometric variables creates complexities in the analysis and interpretation of independent associations of these variables with the development of type 2 diabetes. Principal Component Factor Analysis (PCFA) is the technique to reduce a large number of variables to a smaller number of factors which are more closely associated with antecedent [2-4]. The objective of the present study using principal component factor analysis is to compare the relationship between anthropometric and physiometric components with diabetic males, pre- and post-meno-pausal females in North Indian Punjabi population. The attention has also been given to find out which factors can be used as significant predictors of T2DM.

\section{MATERIALS AND METHODS}

Present study was conducted at the different clinical centres such as Heart Station and Diabetic Clinic, A.P. Hospital and Heart Care Centre, Diabetic Clinic and Research Institute in Amritsar district in the state of Punjab among Punjabi population. Punjabi population may be defined as similar genotype groupings and aggregate of similar cultural practices, life style pattern, social influence and similar ethnic characteristics with Punjabi language speaking and at least reside in Punjab for the last 20 years. A total of 349 type 2 diabetic individuals participated in the baseline examination for the present study which occurred from October 2008 to September 2009. Among total individuals 157 and 192 are males and females respectively whereas, among females 88 and 104 are pre- and post-menopausal. All participants provided written informed consent.

\subsection{Anthropometric Measurements}

Actual age and age on the onset of the disease were recorded from the subject's health card provided by the 
clinical centres. Height, weight, circumferences of waist (WC), hip (HC), arm (AC) and Calf (CC) and four skin fold thickness (biceps, triceps, sub-scapular and supra-iliac) were taken by female author on each individual using standard anthropometric techniques and tools $[5,6]$. Height and weight were measured to the nearest $0.5 \mathrm{~cm}$ and $0.1 \mathrm{~kg}$ respectively. Body mass index (BMI) was calculated for an estimate of overall adiposity using the formula: $\mathrm{BMI}=$ weight $(\mathrm{kg}) /$ height $\left(\mathrm{m}^{2}\right)$. Waist and Hip circumference (WC and $\mathrm{HC}$ ) for an estimate of central obesity [7] were measured to the nearest $0.5 \mathrm{~cm}$ with a steel tape. Waist to hip ratio (WHR) was calculated using the standard formula: WHR $=\mathrm{WC}(\mathrm{cm}) / \mathrm{HC}(\mathrm{cm})$. A Lange skinfold calliper was used to measure the skinfolds to the nearest $0.2 \mathrm{~mm}$. Two subsequent measurements were taken and averages were used in the analysis.

\subsection{Physiometric Measurements}

Left arm blood pressures (first phase systolic and fifth phase diastolic) were taken from each participant with standard mercury sphygmomanometer after a 5 min rest. The average of the two subsequent measurements was used for analysis. All efforts were made to minimize the factors which affect the blood pressure like anxiety, fear, stress, laughing and recent activity [8]. Mean arterial blood pressure (MBP) was calculated for each of the two readings taken for SBP and DBP by using the formula: $\mathrm{MBP}=\mathrm{SBP}+(\mathrm{SBP}-\mathrm{DBP}) / 3$ [9]. The radial artery at the wrist was used to count the pulse. It was counted over one minute. The difference of SBP and DBP was used as pulse pressure.

\section{STATISTICAL ANALYSIS}

Descriptive statistics such as means, standard deviations and coefficient of skewness were calculated for all variables. All statistical analysis including factor analyses were conducted by SPSS (Statistical Package for Social Sciences, version 17.0, SPSS Inc. USA). Each of the anthropometric and physiometric variables is highly inter-correlated with each others and creates a methodological problem for analysis the data. PCFA is used when variables are highly correlated and this multivariate statistical tool able to reduce a large number of inter-correlated variables to a smaller number of principal components which account for most of the variance in the data $[10,11]$. Factor analysis has done on the basis of correlation matrix which helps to understand the amount of association between the variables, factor extraction and orthogonal rotation to make factors easily interpretable. Hence, PCFA was used to extract uncorrelated factors and varimax rotation, which is an orthogonal rotation in which the factors are assumed to act independently, was used in the present study. Factor loadings were equivalent to the correlation coefficients between the variables (rows) and factors (columns). The final factors pattern was interpreted using factor loadings of $\geq 0.4$. Extracted factors or number of factors to be retained was based on eigenvalue criteria $\geq 1.0$. Eigenvalues indicate the amount of variance explained by each factor. A factor with low eigenvalue has a little contribution to explain the variances in the variables and may be ignored. The first and second principal components were identified through largest and second largest amount of variance in the data and so on. Communality is the squared multiple correlation for the variable (as dependent) using as predictors. Hence, the communality estimates is the measure the percent of variance in a given variable explained by all factors. A communality of 0.75 and 0.25 considered large and low respectively. Low communality indicates variables are negligibly related to each other. The probability values less than or equal to 0.05 (two-tailed) were considered to be significant.

\section{RESULTS}

Table 1 presents the mean, standard deviation (SD) and skewness of anthropometric and physiometric variables. All right skewed distributions have converted to a normal distribution by square root transformation whereas; reciprocal transformation is used for left skewed distribution among type 2 diabetic males, pre- and postmenopausal females. The highest mean age for onset of T2DM was found among post-menopausal females $(52.46 \pm 6.22$ years) and the lowest mean age for the onset of disease was found among pre-menopausal females $(36.97 \pm 5.96$ years) as compared to males $(45.19 \pm 7.79$ years). The other highest mean values of important anthropometric indicator such as BMI, hip circumference, biceps skinfold, triceps skinfold and arm circumference were found among diabetic post-menopausal females as compared to males and pre-menopausal females. The diabetic males have higher mean values for WHR and waist circumference. The physiometric variables such as SBP, DBP, pulse rate and pulse pressure have not shown any specific trend among three groups of diabetic subjects. Bivariate correlations of the traits were examined among type 2 diabetic males, pre- and post-menopausal females and are presented in Tables 2 to 4 . Waist circumference, hip circumference, biceps skinfold, triceps skinfold, arm circumference and calf circumference with Weight and BMI; hip circumference, biceps skinfold, triceps skinfold, arm circumference and calf circumference with waist circumference have been found signifi- 
Table 1. Descriptive Statistics of Anthropometric and Physiometric variables among type 2 diabetic male, pre- and post-menopausal females in the present study population $(n=349)$.

\begin{tabular}{|c|c|c|c|c|c|c|c|c|c|}
\hline \multirow{3}{*}{ VARIABLES } & \multirow{2}{*}{\multicolumn{3}{|c|}{ MALE $(\mathbf{N}=157)$}} & \multicolumn{6}{|c|}{ FEMALE $(\mathrm{N}=192)$} \\
\hline & & & & \multicolumn{3}{|c|}{ PRE-MENOPAUSAL $(\mathbf{N}=\mathbf{8 8})$} & \multicolumn{3}{|c|}{ POST-MENOPAUSAL $(\mathrm{N}=104)$} \\
\hline & MEAN & SD & $\begin{array}{l}\text { SKEW- } \\
\text { NESS }\end{array}$ & MEAN & SD & SKEWNESS & MEAN & SD & $\begin{array}{l}\text { SKEW- } \\
\text { NESS }\end{array}$ \\
\hline Onset age (yrs) & 45.197 & 7.792 & 0.836 & 36.97 & 5.96 & -0.28 & 52.46 & 6.22 & 1.02 \\
\hline Height (cm) & 169.131 & 8.582 & -5.437 & 154.95 & 5.599 & 0.19 & 155.80 & 6.95 & 1.57 \\
\hline Weight (Kg) & 76.697 & 6.976 & 0.533 & 63.52 & 7.55 & 0.36 & 67.70 & 8.20 & 0.43 \\
\hline $\operatorname{BMI}\left(\mathrm{kg} / \mathrm{m}^{2}\right)$ & 26.555 & 3.870 & 0.370 & 26.25 & 3.34 & 0.21 & 28.00 & 5.60 & 0.62 \\
\hline WHR & 0.941 & 0.0732 & 0.586 & 0.88 & 0.07 & 0.75 & 0.87 & 0.07 & -0.17 \\
\hline Waist circumference $(\mathrm{cm})$ & 96.014 & 8.512 & 0.267 & 87.23 & 9.90 & 0.597 & 90.26 & 9.32 & 0.49 \\
\hline Hip circumference $(\mathrm{cm})$ & 101.94 & 9.056 & 0.242 & 99.18 & 9.59 & 0.68 & 103.07 & 10.97 & 0.52 \\
\hline Biceps skinfold (mm) & 10.806 & 5.968 & 1.745 & 13.11 & 5.05 & 0.395 & 13.26 & 5.58 & 1.03 \\
\hline Triceps Skinfold (mm) & 14.005 & 5.053 & 3.201 & 17.12 & 5.19 & 0.299 & 17.54 & 6.76 & 0.58 \\
\hline Subscapular skinfold (mm) & ------- & ------- & -------- & 24.94 & 4.88 & -0.39 & 26.66 & 5.399 & 0.04 \\
\hline Supra-iliac skinfold (mm) & ------- & -------- & -------- & 21.37 & 3.82 & -0.14 & 24.37 & 4.43 & 0.10 \\
\hline Arm Circumference (cm) & 27.838 & 3.192 & 0.282 & 27.27 & 3.39 & 0.18 & 28.20 & 3.90 & 0.87 \\
\hline $\begin{array}{l}\text { Systolic blood pressure } \\
\text { (mmHg) }\end{array}$ & 124.656 & 9.266 & 1.802 & 121.72 & 8.82 & 0.86 & 124.95 & 9.49 & 0.798 \\
\hline $\begin{array}{l}\text { Diastolic blood pressure } \\
\text { (mmHg) }\end{array}$ & 80.083 & 10.475 & 1.090 & 79.55 & 9.93 & -0.12 & 79.70 & 9.81 & 1.04 \\
\hline $\begin{array}{l}\text { Mean Blood Pressure } \\
(\mathrm{mmHg})\end{array}$ & 95.924 & 9.384 & 2.081 & 93.56 & 9.67 & 0.57 & 96.14 & 8.27 & 2.695 \\
\hline Pulse Rate & 83.229 & 8.471 & 0.251 & 85.10 & 8.85 & 0.12 & 83.98 & 8.24 & 0.15 \\
\hline Pulse Pressure & 44.790 & 7.232 & 1.660 & 42.27 & 7.58 & 0.77 & 46.41 & 7.08 & 1.296 \\
\hline
\end{tabular}

Significant at least at $\mathrm{p} \leq 0.05$

$\mathrm{SD}=$ Standard Deviation

cantly associated at least 5\% level $(\mathrm{p}<0.05)$ among all diabetic males, pre- and post-menopausal females. SBP and DBP were found to be significantly associated with weight, BMI, waist circumference, hip circumference, biceps skinfold and triceps skinfold at least 5\% level (p $<0.05$ ) among diabetic pre- and post-menopausal females. Whereas, WHR was found significantly associated $(\mathrm{p}<0.05)$ with other anthropometric variables among only type 2 diabetic males.

The comparison of factor loading pattern of six factors (components) is presented in Table $\mathbf{5}$ among diabetic males, pre- and post-menopausal females. Only variables with factor loading greater than or equal to 0.4 were considered for present interpretation among three groups. After Varimax rotation, weight, BMI, waist circumference, hip circumference, arm and calf circumferences are relatively large and positively loaded $(>0.7)$ on factor 1 among males, pre- and post-menopausal females. However, on factor 1 highest loading was found in weight $(0.944)$ for males, hip circumference $(0.883)$ for pre-menopausal females and BMI (0.940) for postmenopausal females. The physiometric variables such as SBP, DBP and pulse pressure are grouped together and loaded positively on factor 2 among three groups. Maximum loading has found for SBP $(>0.90)$ on factor 2 for all three groups, whereas loading of DBP for this factor is just above the cut-off value (0.4) for postmenopausal females. Both mean age (actual age and onset age of type 2 diabetes) have found maximum loading (0.952 and 0.940) on factor 3 among males, whereas, skinfold thickness (biceps, triceps, sub-scapular and supra-iliac) grouped together and loaded significantly among pre- and post-menopausal females. Only triceps skinfold and biceps skinfold among males have positive loading on factor 4 , whereas, actual mean age and the mean age of onset of the disease have grouped for higher positive loading among pre- and post-menopausal females. Only WHR has positive loading on factor 5 among males, whereas, WHR and waist circumference for pre-menopausal females and WHR, height and waist circumference for post-menopausal females have positive loading on this factor. However WHR has maximum positive loading $(\geq 0.80)$ among pre- and post-menopausal females but for males it is just above cut-off value (0.55). Only Height and pulse rate have positive loading on factor 6 among pre- and post-menopausal female whereas, all variables are extracted on this factor among males. The five factors explained $79 \%$ of the total variance among males in which the first two factors cumulatively explained $54 \%$ of the total variance. Whereas, the six factors explained $78 \%$ of the total variance among pre- and post-menopausal females in which first two factors cumulatively explained $48 \%$ and $49 \%$ of the total variance respectively. The eigenvalue of the first two factors have also been seen maximum among males, pre- and post-menopausal females. The 
Table 2. Inter-correlation matrix of selected anthropometric variables among males of Type 2 Diabetes Mellitus (T2DM).

\begin{tabular}{|c|c|c|c|c|c|c|c|c|c|c|c|c|c|c|c|c|c|}
\hline $\begin{array}{l}\text { VARI- } \\
\text { ABLES }\end{array}$ & $\begin{array}{l}\text { Age } \\
\text { (yrs) }\end{array}$ & $\begin{array}{c}\text { OA } \\
\text { (yrs) }\end{array}$ & $\begin{array}{c}\mathrm{Ht} \\
(\mathrm{cm})\end{array}$ & $\begin{array}{c}\text { Wt } \\
(\mathrm{Kg})\end{array}$ & $\begin{array}{c}\text { BMI } \\
\left(\mathrm{kg} / \mathrm{m}^{2}\right)\end{array}$ & WHR & $\begin{array}{l}\text { WC } \\
(\mathrm{cm})\end{array}$ & $\begin{array}{l}\mathrm{HC} \\
(\mathrm{cm})\end{array}$ & $\begin{array}{l}\text { BSkn } \\
(\mathbf{m m})\end{array}$ & $\begin{array}{l}\text { T Skn } \\
(\mathbf{m m})\end{array}$ & $\begin{array}{c}\mathrm{AC} \\
(\mathrm{cm})\end{array}$ & $\begin{array}{l}\mathrm{CC} \\
(\mathrm{cm})\end{array}$ & $\begin{array}{c}\text { SBP } \\
(\mathrm{mmHg})\end{array}$ & $\begin{array}{c}\text { DBP } \\
(\mathrm{mmHg})\end{array}$ & $\begin{array}{c}\text { MBP } \\
(\mathbf{m m H g})\end{array}$ & PR & PP \\
\hline Age (yrs) & & $.81 *$ & -.05 & -.06 & -.007 & .03 & .05 & .03 & -.003 & -.08 & -.04 & -.09 & -.05 & -.05 & -.10 & -.12 & .02 \\
\hline $\begin{array}{c}\text { Onset age } \\
\text { (yrs) }\end{array}$ & & & -.03 & .09 & .16 & .09 & .17 & .16 & .09 & .01 & .10 & .04 & -.08 & -.005 & -0.12 & -.13 & -.06 \\
\hline Height (cm) & & & & .09 & -.15 & -.12 & -.06 & -.007 & -.17 & -.27 & -.02 & -.03 & -.007 & -.07 & -0.04 & -.04 & .03 \\
\hline Weight (Kg) & & & & & $.91^{*}$ & $.48^{*}$ & $.87 *$ & $.84 *$ & $.58 *$ & $.40^{*}$ & $.73 *$ & $.73 *$ & .08 & .18 & 0.11 & -.11 & .05 \\
\hline BMI $\left(\mathrm{kg} / \mathrm{m}^{2}\right)$ & & & & & & $.50^{*}$ & $.85^{*}$ & $.80 *$ & $.57 *$ & $.46^{*}$ & $.73^{*}$ & $.73 *$ & .07 & .18 & 0.11 & -.12 & .03 \\
\hline WHR & & & & & & & $.68^{*}$ & $.20 *$ & $.33^{*}$ & $.40^{*}$ & $.42 *$ & $.34 *$ & .03 & .12 & 0.04 & -.19 & -.001 \\
\hline $\begin{array}{l}\text { Waist cir- } \\
\text { cumference } \\
(\mathrm{cm})\end{array}$ & & & & & & & & $.82 *$ & $.62 *$ & $.45^{*}$ & $.75^{*}$ & $.70 *$ & .01 & .13 & 0.05 & $-.20^{*}$ & -.004 \\
\hline $\begin{array}{l}\text { Hip cir- } \\
\text { cumference } \\
(\mathrm{cm})\end{array}$ & & & & & & & & & $.60^{*}$ & $.32 *$ & $.70^{*}$ & $.70 *$ & -.006 & .09 & 0.05 & -.09 & -.009 \\
\hline $\begin{array}{l}\text { Biceps skin- } \\
\text { fold }(\mathbf{m m})\end{array}$ & & & & & & & & & & $.72 *$ & $.64 *$ & $.50^{*}$ & .003 & .07 & -0.02 & -.13 & .011 \\
\hline $\begin{array}{l}\text { Triceps } \\
\text { Skinfold } \\
(\mathbf{m m})\end{array}$ & & & & & & & & & & & $.46^{*}$ & $.32 *$ & -.04 & .04 & -0.04 & -.13 & -.03 \\
\hline $\begin{array}{l}\text { Arm Cir- } \\
\text { cumference } \\
(\mathrm{cm})\end{array}$ & & & & & & & & & & & & $.68^{*}$ & .03 & .13 & 0.06 & -.13 & -.005 \\
\hline $\begin{array}{c}\text { Calf cir- } \\
\text { cumference } \\
(\mathbf{c m}) \\
\text { Systolic }\end{array}$ & & & & & & & & & & & & & .001 & .04 & 0.01 & -.10 & -.001 \\
\hline $\begin{array}{c}\text { blood pres- } \\
\text { sure } \\
\text { (mmHg) } \\
\text { Diastolic }\end{array}$ & & & & & & & & & & & & & & .73 & $0.86^{*}$ & .08 & $.90^{*}$ \\
\hline $\begin{array}{c}\text { blood pres- } \\
\text { sure } \\
(\mathrm{mmHg})\end{array}$ & & & & & & & & & & & & & & & $0.80^{*}$ & -.004 & $.40 *$ \\
\hline $\begin{array}{l}\text { Mean Blood } \\
\text { Pressure } \\
\text { (mmHg) }\end{array}$ & & & & & & & & & & & & & & & & .053 & $.67 *$ \\
\hline Pulse Rate & & & & & & & & & & & & & & & & & $.09 *$ \\
\hline $\begin{array}{l}\text { Pulse Pres- } \\
\text { sure }\end{array}$ & & & & & & & & & & & & & & & & & \\
\hline
\end{tabular}

Significant at least at $\mathrm{p} \leq 0.05$; $\mathrm{OA}=$ Onset age, $\mathrm{yrs}=$ years, $\mathrm{Ht}=$ Height, $\mathrm{Wt}=$ Weight, $\mathrm{BMI}=$ Body Mass Index, WHR $=$ Waist Hip Ratio, WC $=$ Waist Circumference, $\mathrm{HC}=$ Hip Circumference, $\mathrm{BS}=$ Biceps skinfold, TS $=$ Triceps skinfold, $\mathrm{SS}=$ Sub-scapular skinfold, $\mathrm{SiS}=\mathrm{Supra-iliac}$ skinfold, $\mathrm{AC}=$ Arm Circumference, $\mathrm{CC}=$ Calf Circumference, $\mathrm{SBP}=$ Systolic Blood Pressure, $\mathrm{DBP}=$ Diastollic Blood Pressure, $\mathrm{MBP}=\mathrm{Mean} \mathrm{Blood}$ Pressure, $\mathrm{PR}=$ Pulse Rate, $\mathrm{PP}=$ Pulse Pressure.

common greater communality estimates $(>0.70)$ have found on age, onset age of disease, weight, BMI, waist circumference, hip circumference, triceps skinfold, SBP among three groups. WHR has maximum communality estimates among pre- and post-menopausal females.

\section{DISCUSSION}

The present quantitative analysis have shown that which of the anthropometric and physiometric traits (BMI, WHR, weight, waist circumference, hip circumference, skinfolds, SBP, DBP, and pulse pressure) are more closely associated and act as a good predictors for fur- ther risk among three groups of T2DM individuals such as males, pre- and post-menopausal females in North Indian Punjabi population. The present study also provides through PCFA among three groups that which of the traits would require more attention to clinicians for raised risk of T2DM.

The many previous studies suggested that obesity, overweight, glucose intolerance, hypertension and elevated blood pressures are closely associated with T2DM [1218].The present analysis showed a common association of BMI, WHR, waist circumference, hip circumference and subcutaneous fat with T2DM incidence among males, pre-and post-menopausal females. 
Table 3. Inter-correlation matrix of selected variables among pre-menopausal females of Type 2 Diabetes Mellitus (T2DM).

\begin{tabular}{|c|c|c|c|c|c|c|c|c|c|c|c|c|c|c|c|c|c|c|}
\hline VARIABLES $\begin{array}{l}\text { Age } \\
(\mathrm{yrs})\end{array}$ & $\begin{array}{c}\mathrm{OA} \\
(\mathrm{yrs})\end{array}$ & $\begin{array}{c}\mathrm{Ht} \\
(\mathrm{cm})\end{array}$ & $\begin{array}{l}\text { Wt } \\
(\mathbf{K g})\end{array}$ & $\underset{\left(\mathrm{kg} / \mathrm{m}^{2}\right)}{\mathrm{BMI}}$ & WHR & $\begin{array}{l}\text { WC } \\
\text { (cm) }\end{array}$ & $\begin{array}{l}\mathrm{HC} \\
(\mathrm{cm})\end{array}$ & $\begin{array}{l}\text { BSkn } \\
(\mathrm{mm})\end{array}$ & $\begin{array}{l}\text { T Skn } \\
(\mathrm{mm})\end{array}$ & $\begin{array}{l}\text { SS } \\
(\mathrm{mm})(\end{array}$ & $\begin{array}{c}\mathrm{SiS} \\
(\mathrm{mm})\end{array}$ & $\begin{array}{l}\mathrm{AC} \\
(\mathrm{cm})\end{array}$ & $\begin{array}{l}\mathrm{CC} \\
(\mathrm{cm})(\end{array}$ & $\begin{array}{c}\text { SBP } \\
(\mathrm{mmHg})\end{array}$ & $\begin{array}{c}\text { DBP } \\
(\mathbf{m m H g})\end{array}$ & $\begin{array}{c}\text { MBP } \\
(\mathrm{mmHg})\end{array}$ & PR & PP \\
\hline Age (yrs) & $.54 *$ & -.08 & -.05 & -.14 & .10 & .08 & .03 & .05 & .07 & .06 & .04 & -.09 & -.14 & .13 & -.04 & .02 & -.06 & .22 \\
\hline OA (yrs) & & -.03 & -.03 & -.06 & -.05 & -.03 & .02 & -.02 & .08 & -.006 & .19 & -.04 & .06 & .17 & .10 & .10 & .05 & .19 \\
\hline Ht (cm) & & & .30 & -.08 & .10 & .06 & .0097 & .013 & .04 & .16 & .06 & .15 & .09 & .02 & .03 & .03 & .22 & .006 \\
\hline Wt (Kg) & & & & $.83 *$ & .15 & $.80^{*}$ & $.82 *$ & $.53 *$ & .42 & $.36^{*}$ & $.50 *$ & $.70 *$ & $.51 *$ & .17 & .11 & .19 & -.06 & .13 \\
\hline $\operatorname{BMI}\left(\mathrm{kg} / \mathrm{m}^{2}\right)$ & & & & & .08 & $.68^{*}$ & $.73 *$ & $.58^{*}$ & .45 & $.34 *$ & $.43 *$ & $.68^{*}$ & $.61^{*}$ & .16 & .09 & .18 & -.13 & .15 \\
\hline WHR & & & & & & $.51^{*}$ & -.16 & .009 & -.12 & .10 & .14 & -.03 & -.02 & -.05 & -.02 & .007 & .04 & -.08 \\
\hline WC(cm) & & & & & & & $.75^{*}$ & $.48^{*}$ & $.30^{*}$ & $.22 *$ & $.42 *$ & $.58^{*}$ & $.46^{*}$ & .18 & $.20^{*}$ & $.24 *$ & -.08 & .08 \\
\hline $\mathrm{HC}(\mathrm{cm})$ & & & & & & & & $.56^{*}$ & $.45^{*}$ & $.21 *$ & $.40^{*}$ & $.69^{*}$ & $.53^{*}$ & $.24^{*}$ & $.24 *$ & $.28^{*}$ & -.14 & .14 \\
\hline BS (mm) & & & & & & & & & $.80^{*}$ & $.45^{*}$ & $.46^{*}$ & $.66^{*}$ & $.49 *$ & .15 & .03 & .099 & $-.30^{*}$ & .19 \\
\hline T S (mm) & & & & & & & & & & $.38^{*}$ & $.36^{*}$ & $.54^{*}$ & $.38 *$ & .13 & -.07 & .04 & $-.25 *$ & .25 \\
\hline SS (mm) & & & & & & & & & & & $.66^{*}$ & $.24 *$ & .16 & -.05 & -.05 & -.06 & -.19 & -.03 \\
\hline $\mathrm{SiS}(\mathrm{mm})$ & & & & & & & & & & & & $.35^{*}$ & $.31 *$ & .12 & .09 & .10 & -.05 & .10 \\
\hline $\mathrm{AC}(\mathrm{cm})$ & & & & & & & & & & & & & $.66^{*}$ & .008 & -.03 & .03 & -.13 & .02 \\
\hline $\mathrm{CC}(\mathrm{cm})$ & & & & & & & & & & & & & & .008 & .04 & .05 & -.14 & -.008 \\
\hline SBP (mmHg) & & & & & & & & & & & & & & & $.71 *$ & $.91 *$ & -.09 & $.87 *$ \\
\hline $\begin{array}{l}\text { DBP } \\
(\mathrm{mmHg})\end{array}$ & & & & & & & & & & & & & & & & $.85^{*}$ & .09 & $.29^{*}$ \\
\hline $\begin{array}{c}\text { MBP } \\
(\mathbf{m m H g})\end{array}$ & & & & & & & & & & & & & & & & & -.009 & $.65^{*}$ \\
\hline P R & & & & & & & & & & & & & & & & & & -.17 \\
\hline $\mathbf{P} \mathbf{P}$ & & & & & & & & & & & & & & & & & & \\
\hline
\end{tabular}

Significant at least at $\mathrm{p} \leq 0.05 ; \mathrm{OA}=$ Onset age, $\mathrm{yrs}=$ years, $\mathrm{Ht}=$ Height, $\mathrm{Wt}=$ Weight, $\mathrm{BMI}=$ Body Mass Index, WHR $=$ Waist Hip Ratio, WC $=$ Waist Circumference, $\mathrm{HC}=$ Hip Circumference, $\mathrm{BS}=$ Biceps skinfold, TS = Triceps skinfold, SS = Sub-scapular skinfold, SiS $=$ Supra-iliac skinfold, $\mathrm{AC}=$ Arm Circumference, $\mathrm{CC}=$ Calf Circumference, $\mathrm{SBP}=$ Systolic Blood Pressure, $\mathrm{DBP}=$ Diastolic Blood Pressure, $\mathrm{MBP}=\mathrm{Mean} \mathrm{Blood}$ Pressure, $\mathrm{PR}=$ Pulse Rate, $\mathrm{PP}=$ Pulse Pressure.

Table 4. Inter-correlation matrix of variables among post-menopausal females of Type 2 Diabetes Mellitus (T2DM).

\begin{tabular}{|c|c|c|c|c|c|c|c|c|c|c|c|c|c|c|c|c|c|c|c|}
\hline VARIABLES & $\begin{array}{l}\text { Age } \\
\text { (yrs) }\end{array}$ & $\begin{array}{c}\text { OA } \\
\text { (yrs) }\end{array}$ & $\begin{array}{c}\mathrm{Ht} \\
(\mathrm{cm})\end{array}$ & $\begin{array}{l}\text { Wt } \\
(\mathrm{Kg})\end{array}$ & $\begin{array}{c}\text { BMI } \\
\left(\mathrm{kg} / \mathrm{m}^{2}\right)\end{array}$ & WHR & $\begin{array}{l}\text { WC } \\
(\mathrm{cm})\end{array}$ & $\begin{array}{l}\text { HC I } \\
(\mathrm{cm})(\end{array}$ & $\begin{array}{l}\text { BSkn } \\
(\mathbf{m m})\end{array}$ & $\begin{array}{l}\text { T Skn } \\
(\mathrm{mm})\end{array}$ & $\begin{array}{c}\text { SS } \\
(\mathbf{m m})(\end{array}$ & $\begin{array}{c}\mathrm{SiS} \\
(\mathbf{m m})\end{array}$ & $\begin{array}{l}\mathrm{AC} \\
(\mathrm{cm})\end{array}$ & $\begin{array}{l}\mathrm{CC} \\
(\mathrm{cm})(\end{array}$ & $\begin{array}{c}\text { SBP } \\
\text { mmHg) }\end{array}$ & $\begin{array}{c}\text { DBP } \\
(\mathrm{mmHg})(\end{array}$ & $\begin{array}{l}\text { MBP } \\
(\mathrm{mmHg})\end{array}$ & PR & PP \\
\hline Age (yrs) & & $.73^{*}$ & -.12 & .007 & .06 & -.01 & .17 & .15 & -.08 & -.02 & $-.20^{*}$ & -.07 & -.04 & .03 & .06 & -.07 & .14 & .15 & .09 \\
\hline OA (yrs) & & & -.08 & -.03 & .006 & .04 & .07 & .03 & -.04 & .04 & -.09 & -.05 & -.08 & -.02 & .06 & .099 & .16 & .15 & .005 \\
\hline Ht (cm) & & & & .19 & $-.23^{*}$ & .17 & .06 & -.05 & .04 & .10 & .03 & -.08 & -.09 & -.02 & -.15 & -.07 & -.11 & -.03 & -.19 \\
\hline Wt (Kg) & & & & & $.90 *$ & .18 & $.80^{*}$ & $.83^{*}$ & $.53^{*}$ & $.40^{*}$ & $.54 *$ & $.52 *$ & $.76^{*}$ & $.74 *$ & .13 & .18 & .10 & .05 & -.005 \\
\hline BMI $\left(\mathrm{kg} / \mathrm{m}^{2}\right)$ & & & & & & .07 & $.77^{*}$ & $.85^{*}$ & $.50 *$ & $.35^{*}$ & $.52 *$ & $.55^{*}$ & $.80 *$ & $.74 *$ & .20 & $.20 *$ & .16 & .08 & .09 \\
\hline WHR & & & & & & & $.47^{*}$ & -.08 & .10 & .10 & .10 & .03 & .01 & -.02 & .002 & -.09 & .10 & -.03 & .05 \\
\hline WC(cm) & & & & & & & & $.796^{*}$ & $.53^{*}$ & $.42 *$ & $.495^{*}$ & $.46^{*}$ & $.65^{*}$ & $.62 *$ & .19 & .083 & .20 & .10 & .15 \\
\hline $\mathrm{HC}(\mathrm{cm})$ & & & & & & & & & $.50 *$ & 1.0 & $.55^{*}$ & $.53 *$ & $.77 *$ & $.75^{*}$ & .16 & .12 & .14 & .05 & .09 \\
\hline BS (mm) & & & & & & & & & & $.78^{*}$ & $.50 *$ & $.47 *$ & $.44 *$ & $.44 *$ & .06 & .05 & .009 & .013 & .06 \\
\hline T S (mm) & & & & & & & & & & & $.44 *$ & $.37 *$ & $.33^{*}$ & $.33^{*}$ & .03 & -.05 & .01 & .013 & .10 \\
\hline SS (mm) & & & & & & & & & & & & $.60^{*}$ & $.49^{*}$ & $.43^{*}$ & -.09 & -.02 & -.15 & -.002 & -.12 \\
\hline $\mathrm{SiS}(\mathbf{m m})$ & & & & & & & & & & & & & $.48^{*}$ & $.49^{*}$ & .18 & .12 & .03 & -.03 & .12 \\
\hline $\mathrm{AC}(\mathrm{cm})$ & & & & & & & & & & & & & & $.69^{*}$ & .12 & $.21^{*}$ & .12 & .05 & .007 \\
\hline $\mathrm{CC}(\mathrm{cm})$ & & & & & & & & & & & & & & & $.20^{*}$ & .16 & .12 & .06 & .14 \\
\hline SBP (mmHg) & & & & & & & & & & & & & & & & $.59 *$ & $.72 *$ & .13 & $.86^{*}$ \\
\hline DBP (mmHg) & & & & & & & & & & & & & & & & & $.56^{*}$ & .13 & .16 \\
\hline MBP (mmHg) & & & & & & & & & & & & & & & & & & .003 & $.53^{*}$ \\
\hline PR & & & & & & & & & & & & & & & & & & & .06 \\
\hline PP & & & & & & & & & & & & & & & & & & & \\
\hline
\end{tabular}

Significant at least at $\mathrm{p} \leq 0.05 ; \mathrm{OA}=$ Onset age, $\mathrm{yrs}=$ years, $\mathrm{Ht}=$ Height, $\mathrm{Wt}=$ Weight, $\mathrm{BMI}=$ Body Mass Index, WHR $=$ Waist Hip Ratio, WC $=$ Waist Circumference, $\mathrm{HC}=$ Hip Circumference, $\mathrm{BS}=$ Biceps skinfold, $\mathrm{TS}=$ Triceps skinfold, $\mathrm{SS}=$ Sub-scapular skinfold, $\mathrm{SiS}=\mathrm{Supra-iliac}$ skinfold, $\mathrm{AC}=$ Arm Circumference, $\mathrm{CC}=$ Calf Circumference, $\mathrm{SBP}=$ Systolic Blood Pressure, $\mathrm{DBP}=$ Diastolic Blood Pressure, MBP $=$ Mean Blood Pressure, $\mathrm{PR}=$ Pulse Rate, $\mathrm{PP}=$ Pulse Pressure 
Table 5. Comparison of factor loadings by principal component analysis with Varimax rotation and communalities of the risk factors among type 2 diabetic male, pre-menopausal and post-menopausal females $(n=349)$.

\begin{tabular}{|c|c|c|c|c|c|c|c|c|c|c|c|c|c|c|c|c|c|c|c|c|c|}
\hline \multirow[t]{2}{*}{ VARIABLES } & \multicolumn{3}{|c|}{ FACTOR 1} & \multicolumn{3}{|c|}{ FACTOR 2} & \multicolumn{3}{|c|}{ FACTOR 3} & \multicolumn{3}{|c|}{ FACTOR 4} & \multicolumn{3}{|c|}{ FACTOR 5} & \multicolumn{3}{|c|}{ FACTOR 6} & \multicolumn{3}{|c|}{$\begin{array}{c}\text { COMMU- } \\
\text { NALITY } \\
\text { ESTIMATES }\end{array}$} \\
\hline & $\mathbf{M}$ & PRF & POF & $\mathbf{M}$ & PRF & POF & $\mathbf{M}$ & PRF & POF & M & PRF & POF & M & PRF & POF & $\mathbf{M}$ & PRF & POF & M & $\begin{array}{c}\text { PR } \\
\text { F }\end{array}$ & $\begin{array}{c}\text { PO } \\
\text { F }\end{array}$ \\
\hline Age (yrs) & -.046 & -.083 & .042 & -.025 & .038 & .041 & .952 & .057 & -.072 & .008 & .860 & .930 & .053 & .136 & -.021 & ---- & -.134 & .010 & .912 & .787 & .874 \\
\hline Onset age (yrs) & .114 & .013 & -.035 & -.054 & .105 & .048 & .940 & .021 & .021 & .015 & .868 & .873 & .054 & -.097 & .006 & ---- & .111 & .168 & .902 & .787 & .794 \\
\hline Height (cm) & .056 & .041 & -.099 & -.035 & .030 & -.217 & -.080 & .303 & .104 & -.827 & -.084 & -.196 & .118 & .007 & .652 & ---- & .726 & .404 & .709 & .629 & .695 \\
\hline Weight (Kg) & .944 & .833 & .903 & .091 & .117 & .017 & -.147 & .260 & .160 & -.043 & -.042 & -.029 & .102 & .255 & .241 & --- & .173 & .129 & .912 & .872 & .917 \\
\hline WHR & .432 & -.029 & .072 & .061 & -.060 & .092 & .007 & .107 & .034 & .278 & .025 & .080 & .550 & .913 & .831 & --- & .006 & -.296 & .569 & .849 & .799 \\
\hline Waist circumference(cm) & .900 & .735 & .796 & .033 & .133 & .139 & .078 & .087 & .215 & .117 & .039 & .182 & .253 & .620 & .412 & --- & -.030 & -.072 & .894 & .953 & .908 \\
\hline Hip circumference $(\mathrm{cm})$ & .911 & .883 & .903 & .002 & .202 & .048 & .088 & .060 & .184 & -.031 & .039 & .103 & -.088 & .019 & -.064 & --- & -.037 & .027 & .846 & .827 & .867 \\
\hline Biceps skinfold (mm) & .663 & .633 & .419 & -.020 & .067 & .025 & -.002 & .527 & .794 & .473 & .043 & -.046 & .106 & -.125 & .064 & ---- & -.271 & .045 & .675 & .775 & .814 \\
\hline Triceps Skinfold (mm) & .429 & .511 & .245 & -.051 & .033 & .021 & -.110 & .540 & .880 & .680 & .114 & .045 & .245 & -.267 & .103 & --- & -.257 & .036 & .722 & .704 & .848 \\
\hline Subscapular skinfold (mm) & -- & .137 & .571 & -- & -.071 & -.197 & -- & .871 & .469 & --- & -.030 & -.179 & -- & .103 & .005 & --- & .032 & -.037 & --- & .795 & .619 \\
\hline Supra-iliac skinfold (mm) & -- & .346 & .577 & -- & .079 & .091 & -- & .684 & .413 & --- & .125 & -.104 & -- & .202 & -.143 & --- & .105 & -.107 & ----- & .661 & .554 \\
\hline Arm Circumference (cm) & .838 & .863 & .871 & .030 & -.071 & .037 & -.006 & .175 & .077 & .115 & -.041 & -.078 & .113 & -.096 & -.053 & --- & .014 & .070 & .729 & .792 & .780 \\
\hline Calf circumference (cm) & .837 & .763 & .811 & -.025 & -.046 & .103 & -.062 & .048 & .141 & -.011 & -.021 & -.006 & .002 & -.135 & -.053 & --- & .031 & .057 & .705 & .606 & .694 \\
\hline $\begin{array}{l}\text { Diastolic blood pressure } \\
(\mathrm{mmHg})\end{array}$ & .097 & .076 & .190 & .823 & .817 & .579 & -.009 & -.117 & -.163 & .061 & -.063 & -.135 & .086 & .082 & -.084 & --- & .178 & .539 & 698 & .729 & .714 \\
\hline $\begin{array}{l}\text { Mean Blood Pressure } \\
(\mathrm{mmHg})\end{array}$ & .035 & .104 & .102 & .934 & .965 & .833 & -.082 & -.043 & -.115 & -.007 & -.014 & .103 & -.003 & .046 & .112 & ---- & .046 & .108 & .880 & .948 & .752 \\
\hline Pulse Rate & -.049 & -.084 & .028 & .060 & -.032 & .035 & -.097 & -.254 & .062 & .088 & .065 & .235 & -.862 & -.005 & -.059 & --- & .752 & .642 & .769 & .642 & .477 \\
\hline Pulse Pressure & .007 & .015 & -.032 & .814 & .767 & .846 & .020 & .165 & .178 & -.029 & .207 & .066 & -.084 & -040 & -.060 & --- & -.209 & -.215 & .671 & .721 & .802 \\
\hline Eigenvalue & 6.016 & 5.917 & 6.408 & 3.190 & 3.159 & 2.876 & 1.837 & 1.762 & 1.804 & 1.318 & 1.574 & 1.444 & 1.034 & 1.228 & 1.201 & --- & 1.204 & 1.034 & -- & -- & -- \\
\hline Variance Explained(\%) & 35.389 & 31.140 & 33.726 & 18.767 & 16.626 & 15.135 & 10.806 & 9.274 & 9.496 & 7.756 & 8.286 & 7.601 & 6.084 & 6.464 & 6.322 & --- & 6.336 & 5.440 & ---- & --- & --- \\
\hline Cumulative Variance(\%) & 35.389 & & & 54157 & 77. & & 64.962 & $\begin{array}{c}57.04 \\
1\end{array}$ & & & & & & & & -- & 78.127 & & --- & --- & --- \\
\hline
\end{tabular}

PCFA is applied to identify the significant association with T2DM among three groups. As far as concern in the North Indian Punjabi population, very little information $[15,19-21]$ to identify the underlying factors/components of the T2DM are available. In this consideration the present work has been undertaken among the males and females (pre- and post-menopausal). PCFA have identified five factors with $79 \%$ explained variance among male diabetic subjects and six factors with $78 \%$ explained variance among pre- and post-menopausal diabetic female subjects.

It is important to note that neither of the anthropometric and physiometric variables equally loaded on all five or six components. Factor 1 is the most diverse among three groups. It could be identified as weight, BMI, waist circumference for males; hip circumference, BMI, weight for pre-menopausal females and BMI, weight, hip circumference for post-menopausal females. However weight for males, hip circumference for pre-menopausal and BMI for post- menopausal females are heavily loaded. The second factor could be identified as SBP and DBP for males and pre-menopausal females and DBP for post-menopausal females. This component is most clearly and heavily loaded. Therefore, among diabetic individuals, males and pre-menopausal females were very closely associated with SBP and DBP whereas, post-menopausal diabetic females were more concerned with DBP only. Among male diabetic subjects factor three was grouped with actual age and age of onset of the disease, whereas, subcutaneous fat was identified as factor three. Factor four could be identified as subcutaneous fat for males, whereas actual age and age of onset of the disease were grouped together for factor four.

Factor five could be identified as WHR for the three groups. However, WHR is heavily loaded for pre- and post-menopausal females. Pulse rate could be identified as factor six for both pre- and post-menopausal females, whereas, no sixth factor is identified for males.

The present factor analysis confirmed that cluster of at least three variables such as, weight, BMI, waist circumference which have identified as factor one explained $35 \%, 31 \%$ and $34 \%$ of the total variance among diabetic males, pre- and post-menopausal females respectively. Therefore, the cluster of weight, BMI and waist circumference could be classified as central obesity and this cluster is equally associated with diabetic males and post-menopausal females. Furthermore, in the present study, the second factor, that is blood pressures explained $19 \%, 17 \%$ and $15 \%$ of the total variance among diabetic males, pre- and post-menopausal females. The blood pressures (SBP and DBP) were positively and significantly associated with diabetic males. Therefore the above two types factors such as, central obesity and blood pressures are more predispose among diabetic males as compared to females. PCFA also confirmed that WHR and pulse pressure are significantly 
associated with diabetic pre- and post-menopausal females as compared to diabetic males. Therefore, it is very difficult to single out of the particular variable which is more associated with male or pre- and postmenopausal females due to the fact that many overlapping variables have found as more than one factor among all the three groups. Further, research with PCFA is required on other Indian ethnic groups to compare the present trend of the study.

\section{ACKNOWLEDGEMENTS}

The authors are greatful to Dr Rohit Kapoor; Dr. A. P. Singh and Dr. Puneet Arora for their co-operation during the data collection. This work is financially supported by University Grants Commission, New Delhi [DRS I (UGC-SAP)].

\section{REFERENCES}

[1] Meigs, J.B. (2000) Invited commentary: Insulin resistance syndrome? Syndrome X? Multiple metabolic syndrome? A syndrome at all? Factor analysis reveals patterns in the fabric of correlated metabolic risk factors. American Journal of Epidemiology, 152(10), 908-911.

[2] Hanley, A.J., Karter, A.J. and Festa, A. (2002) Factor analysis of metabolic syndrome using directly measured insulin sensitivity: The insulin resistance atherosclerosis study. Diabetes, 51(8), 2642-2647.

[3] Ghose, A., Bose, K. and Das Chaudhari, A.B. (2003) Association of food pattern, central obesity measures and metabolic risk factors for coronary heart disease (CHD) in middle aged Bengalee Hindu men, Calcutta, India. Asia Pacific Journal of Clinical Nutrition, 12(2), 166171.

[4] Ghose, A. (2005) Factor analysis of metabolic syndrome among the middle-aged Bengalee Hindu men of Calcutta, India. Diabetes/Metabolism Research and Reviews, 21(1), 58-64.

[5] Singh, I.P. and Bhasin, M.K. (1968) Anthropometry, Kamla Raj Enterprises, Delhi.

[6] Weiner, J.S. and Lourie, J.A. (1981) Practical Human Biology, Academic Press, London.

[7] Lemieux, S., Prud'homme, D., Bouchard, C., Tremblay, A. and Despres, J.P. (1996) A single threshold value of waist girth identifies normal weight and overweight subjects with excess visceral adipose tissue. American Journal of Clinical Nutrition, 64(5), 685-693.

[8] Badaruddoza and Afzal, M. (1999) Age specific differences in blood pressure among inbred and non-inbred North Indian children. Journal of Biosciences, 24(2), 177-184.

[9] Perusse, L., Rice, T., Bouchard, C., Vogler, G.P. and Rao, D.C. (1989) Cardiovascular risk factors in the French Canadian population: Resolution of genetic and familial environmental effects on blood pressure by using extensive information on environmental correlates. American Journal of Human Genetics, 45(2), 240-251.

[10] Truxillo, C. (2003) Multivariate statistical methods: Prac- tical research applications course notes. SAS Institute, USA.

[11] Wu, C.Z., Lin, J.D., Li, J.C., Hsiao, F.C., Hsich, C.H., Kuo, S.W., Hung, Y.J., Lu, C.H., He, C.T. and Pei, D. (2008) Factor analysis of metabolic syndrome using direct measurement of insulin resistance in Chinese with different degrees of glucose tolerance. Indian Journal of Medical Research, 127(1), 336-343.

[12] Singh, R., Shaw, J. and Zimmet, P. (2004) Epidemiology of childhood type 2 diabetes in the developing world. Pediatric Diabetes, 5(3), 154-168.

[13] Li, J.K., Ng, M.C., So, W.Y., Chiu, C.K., Ozaki, R., Tong, P.C., Cockram, C.S. and Chan, J.C. (2006) Phenotypic and genetic clustering of diabetes and metabolic syndrome in Chinese families with type 2 diabetes mellitus. Diabetes/Metabolism Research and Reviews, 22(6), 46-52.

[14] Comuzzie, A.G., Tejero, M.E., Funahash, T., Martin, L.J., Kisseban, A., Takahashi, S., Tanaka, S., Rainwater, D.L., Matsuzawa, Y., MacCluer, J.W. and Blangero, J. (2007) The genes influencing adiponectin levels also influence risk factors for metabolic syndrome and type 2 diabetes. Human Biology, 79(2), 191-200.

[15] Rai, E., Sharma, S., Koul, A., Bhat, A.K., Bhanwer, A.J. and Bamezai, R.N. (2007) Interaction between the UCP2-866G/A, mtDNA 10398G/A and PGClalpha p.Thr394Thr and p.Gly482Ser polymorphisms in type 2 diabetes in North Indian population. Human Genetics, 122(5), 535-540.

[16] Franceschini, N., Almasy, L., MacCluer, J.W., Goring, H.H., Cole, S.A., Diego, V.P., Laston, S., Howard, B.V., Lee, E.T., Best, L.G., Fabsitz, R.R. and North, K.E. (2008) Diabetes-specific genetic effects on obesity traits in American Indian populations: The strong heart family study. BMC Medical Genetics, 9(1), 90-96.

[17] van't Riet, E., Alsseme, M., Nijpels, G. and Dekker, J.M. (2008) Estimating the individual risk of diabetes: Not on the grounds of overweight only. Nederlands Tijdschr Geneeskd, 152(44), 2385-2388.

[18] Dunkley, A.J., Taub, N.A., Davis, M.J., Stone, M.A. and Khunti, K.C. (2009) Is having a family history of type 2 diabetes or cardiovascular disease a predictive factor for metabolic syndrome? Primary Care Diabetes, 3(1), 49-56.

[19] Matharoo, K., Kumar, A., Randhawa, N.K., Arora, P. and Bhanwer, A.J.S. (2006) Angiotensin converting enzyme gene insertion/deletion polymorphism in type 2 diabetes (T2DM) patients from Punjab. Journal of Punjab Academy of Sciences, 3(3), 7-12.

[20] Sanghera, D.K., Bhatti, J.S., Bhatti, G.K., Ralhan, S.K., Wander, G.S., Singh, J.R., Bunker, C.H., Weeks, D.E., Kamboh, M.I. and Ferrell, R.E. (2006) The Khatri Sikh Diabetes Study (SDS): Study design, methodology, sample collection, and initial results. Human Biology, 78(1), 43-63.

[21] Sanghera, D.K., Ortega, L., Han, S., Singh, J., Ralhan, S.K., Wander, G.S., Mehra, N.K., Mulvihill, J.J., Ferrell R.E., Nath, S.K. and Kamboh, M.I. (2008) Impact of nine common type 2 diabetes risk polymorphisms in Asian Indian Sikhs: PPARG2 (Pro12Ala), IGF2BP2, TCF7L2 and FTO variants confer a significant risk. BMC Medical Genetics, 9(1), 59-67. 